\title{
Zoonotic tuberculosis in humans assessed by next-generation sequencing: an 18-month nationwide study in Lebanon
}

\author{
To the Editor:
}

The World Health Organization (WHO) and other international organisations, including the Food and Agriculture Organization of the United Nations, the World Organisation for Animal Health and the International Union Against Tuberculosis and Lung Disease recently called for formally assessing and (re)prioritising the burden of zoonotic tuberculosis (TB) in people, due to Mycobacterium bovis [1, 2]. Its global contribution to human TB, otherwise principally caused by Mycobacterium tuberculosis, might be underestimated [2]. Nationally representative prevalence data are virtually non-existent on continents with the highest presumed burdens, i.e. in Africa and Asia [3].

In addition to causing hard-to-diagnose extrapulmonary $\mathrm{TB}$ more frequently, $M$. bovis is naturally resistant to pyrazinamide [4], a crucial drug for the standard short-course anti-TB therapy. Due to reliability issues, phenotypic susceptibility to pyrazinamide is often not tested [5]. The most commonly used phenotypic and molecular diagnostics, including the WHO-endorsed GeneXpert MTB/RIF test (Cepheid, Sunnyvale, CA, USA), do not differentiate M. bovis from M. tuberculosis [1]. Thus, M. bovis-infected patients may receive inadequate treatment, risking poorer outcomes [6]. Underdiagnosis in people also implies the existence of undetected animal and food sources and zoonotic risks escaping common TB control measures $[1,2]$.

In Europe, patients with M. bovis infection are often African- or southern Mediterranean-born, suggesting regional endemicity $[6,7]$. We determined the prevalence of $M$. bovis-caused TB in a survey including all TB patients reported to the national TB programme over an 18-month period in Lebanon. In addition to its national population and $>1.5$ million Syrian and Palestinian refugees, this Mediterranean country hosts large numbers of migrant workers from Africa and Asia [8]. Many are from Ethiopia [8], where proportions of extrapulmonary TB and $M$. bovis-caused disease among TB patients apparently culminate, reaching $\sim 30 \%$ [9] and 15-30\% (in focal studies; [10]), respectively. We used a novel targeted next-generation sequencing-based assay for extensive drug resistance detection, including resistance to pyrazinamide, and genotypic differentiation between $M$. bovis and M. tuberculosis [11].

This survey was approved by the Azm Center/Lebanese University ethical committee (document CE-EDST-3-2016). Clinical samples were collected from all 1104 different TB patients with presumption of pulmonary or extrapulmonary TB, based on the presence of symptoms and prior tuberculin skin testing or radiological examination in local hospitals for a number of patients, and reported to all national anti-TB centres between June 1, 2016 and November 31, 2017. All of these 1104 patients were tested by at least one of the following assays: solid (Lowenstein-Jensen) and/or liquid (BBL MGIT; Beckton Dickinson, Franklin Lakes, NJ, USA) culturing, GeneXpert MTB/RIF on sputum, and Anyplex MTB/NTM Real-time (Seegene, Seoul, Republic of Korea) on sputum or culture on solid or liquid medium. Out of these 1104, 417 were confirmed as TB positive by GeneXpert and/or Anyplex. Among these 417 patients, 354 were culture positive. Among the 354 corresponding patients, 325 were new TB patients, 22 had a previous TB history, while TB history information was unavailable for seven patients.

Available DNA extracts, obtained from 348 out of 354 primary cultures by using MasterPure ${ }^{\mathrm{TM}}$ DNA Purification Kit (Epicentre, Illumina, Madison, WI, USA), were subjected to targeted sequencing, more

@ERSpublications

In response to recent international calls, this study reveals the nationally representative prevalence of zoonotic tuberculosis in people in a non-high income country, highlighting the need for appropriate diagnostics and treatment of these patients http://bit.ly/2l3ydDh

Cite this article as: El Achkar S, Demanche C, Osman M, et al. Zoonotic tuberculosis in humans assessed by next-generation sequencing: an 18-month nationwide study in Lebanon. Eur Respir J 2020; 55: 1900513 [https://doi.org/10.1183/13993003.00513-2019]. 
tolerant to low DNA integrity (following suboptimal sample transportation from Lebanon to France) than whole-genome sequencing. Briefly, the Deeplex-MycTB assay (Genoscreen, Lille, France) uses a 24-plexed amplification of mycobacterial species identification ( $h$ sp65), genotyping (spoligotyping and phylogenetic single nucleotide polymorphisms (SNPs)) and $18 \mathrm{M}$. tuberculosis complex drug resistance-associated gene targets, including the main pyrazinamide resistance-associated gene $p n c A$ (figure 1). Paired-end amplicon libraries of 150-bp read length (Nextera XT kit; Illumina, San Diego, CA, USA) were sequenced in a single Illumina NextSeq run. Variant calling and genotypic analysis were performed using a parameterised software (GenoScreen).

Among 339 (97.4\%) out of 348 samples with exploitable sequencing data, 11 were concordantly identified as $M$. bovis strains by typical spoligotype signatures (missing spacers 39-43) and the canonical phylogenetic SNP pncA H57D causing natural pyrazinamide resistance [5] (figure 1). No other known drug resistance-associated mutations were detected. Standard 24-locus mycobacterial interspersed repetitive unit-variable-number tandem repeat (MIRU-VNTR) typing [12] followed by MIRU-VNTRplus database identification [13] confirmed $M$. bovis classification in all cases, and identified one additional $M$. bovis infection among the nine samples without sequencing data. A total of 12 (3.4\%) out of 348 patients were thus infected with M. bovis; 11 were new TB patients. The remaining 336 had human-associated TB strains (i.e. M. tuberculosis or M. africanum).

Logistic regression analysis was used to identify independent predictors of $M$. bovis infection. Potential covariates were patient age, sex, treatment outcome, new case versus previously treated case, nationality and extrapulmonary TB. Normal distribution of selected continuous variables was assessed by the Shapiro-Wilk test. The log-linearity assumption being violated for patient age, a piecewise log-linear regression model was considered. The multivariate model was built by minimising Schwarz's bayesian criterion and maximising the c-statistics. The final model was assessed using the Pearson goodness-of-fit test. The two-sided Type I error was set at 5\%. Median (interquartile range) patient age was 40 (22-62) years with $M$. bovis versus 29 (25-38) years with human-associated strains (per-year adjusted odds ratio if age $\geqslant 55$ years 1.14, 95\% CI 1.01-1.28; $\mathrm{p}=0.04)$. Out of the 12 patients with $M$. bovis, five $(41.6 \%)$ had extrapulmonary TB (including gastric TB for a 2-year-old infant), versus eight (2.4\%) out of 336 patients with human-associated strains (adjusted OR 16.9, 95\% CI 3.5-80.7; $\mathrm{p}=0.0004$ ). The single patient who had both pulmonary and extrapulmonary disease had a M. tuberculosis strain. This case was categorised as extrapulmonary when analysing the association between extrapulmonary infection and $M$. bovis or human-associated strains. While Lebanese nationals represented only 86 (25.6\%) out of 336 patients with human-associated strains, they comprised 10 (83.3\%) out of the 12 patients with M. bovis (adjusted OR 6.9, 95\% CI 1.4-35.5; $\mathrm{p}=0.02$ ). The two remaining patients were a Syrian refugee and a Syrian resident. Other covariates, such as the proportion of new TB patients ( $92 \%$ in both M. bovis and human-associated strain categories), were not statistically associated with risk of $M$. bovis infection.

Patients with $M$. bovis were all from different places disseminated over the country, and unique 24-locus MIRU-VNTR types were detected for each isolate, which was unsupportive of interpatient transmission or a common source of infection. Phylogenetic reconstruction indicated that these different strains represent at least two distinct $M$. bovis sublineages, distinguished by, for example, a fabG1 SNP and distinct spoligotype signatures (figure 1). Infections by distinct strains in residents from disseminated places inform the probable existence of multiple zoonotic sources (e.g. infected cattle, unpasteurised dairy produce) unidentified in the country.

None of the 148 migrant patients carried M. bovis, although they originated mainly from Ethiopia $(\mathrm{n}=94)$ and Sudan $(\mathrm{n}=13)$ where zoonotic infection has been documented or presumed and linked to widespread pastoralism [10]. Other migrants were from other high TB incidence countries (e.g. 18 from Bangladesh, nine from the Philippines). While the lack of M. bovis infection among migrant workers could reflect low M. bovis burdens in these countries, it might also partly result from 1) selective recruitment of candidates who manifest no medical issues (thus unlikely to have active TB) by overseas employment agencies in the country of origin; 2) putatively lower risk of progression to disease among patients latently infected with $M$. bovis than with $M$. tuberculosis, due to lower virulence of $M$. bovis [14, 15]; and 3) residency in the country of destination often limited to a few years. More studies in those countries would be needed to investigate this.

In a Dutch longitudinal study, no association was found between patient outcome and the use of standard first-line TB treatment (4-months rifampicin/isoniazid/ethambutol/pyrazinamide, 2-months rifampicin/ isoniazid) instead of a regimen adopted by some countries for treating M. bovis disease (9-months isoniazid/rifampicin, 2-months ethambutol) [6]. Nevertheless, the mortality with $M$. bovis disease was found to be higher relative to $M$. tuberculosis, presumably reflecting more prevalent miliary and central nervous system (CNS) localisation of $M$. bovis. While the $12 \mathrm{M}$. bovis-infected patients in our 
a)

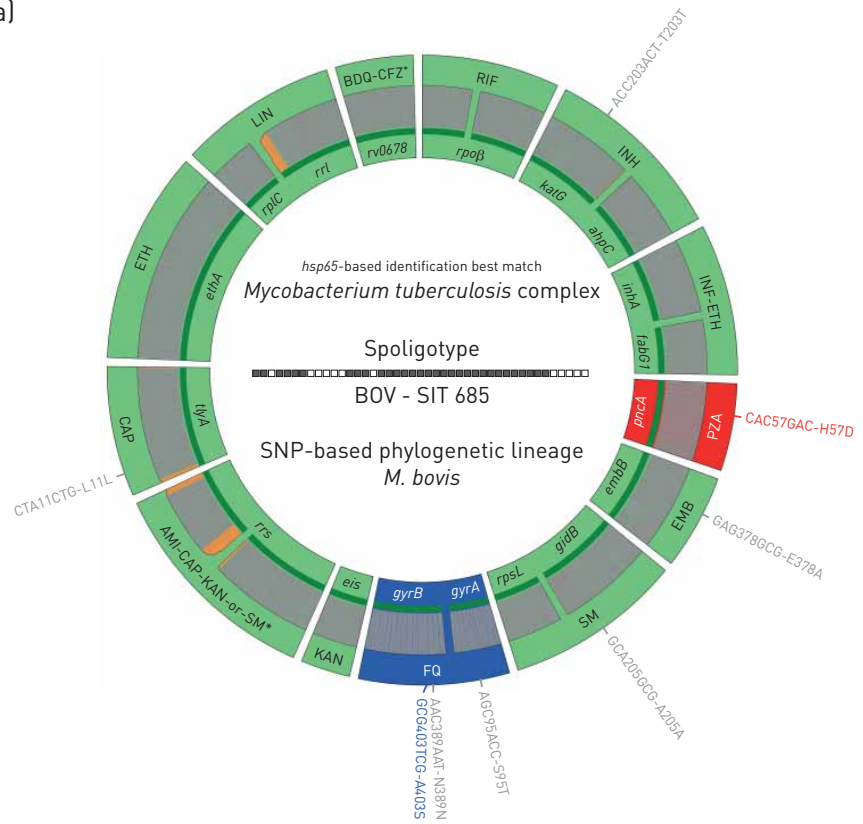

b)

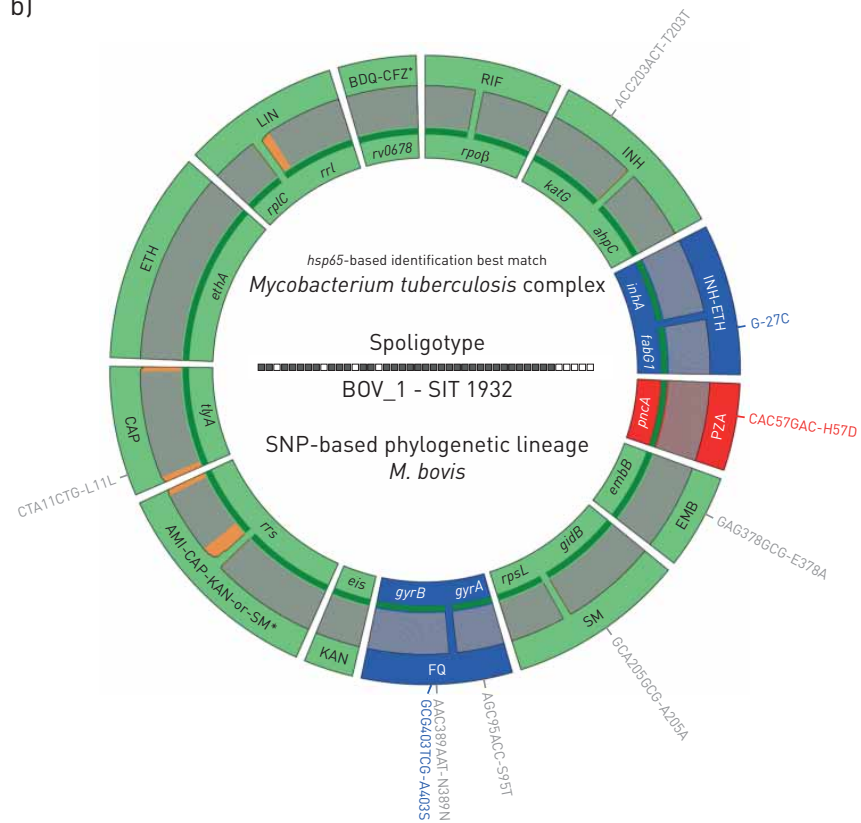

FIGURE 1 Deeplex-MycTB results identifying Mycobacterium bovis-caused zoonotic tuberculosis in patients in Lebanon. Target gene regions are grouped within sectors in a circular map according to the anti-tuberculous drug resistance with which they are associated. The sector in red refers to the pncA region in which the pyrazinamide resistance-causing H57D mutation (shown in red around the circular map) canonically associated with $M$. bovis is detected. Sectors in green or blue refer to regions where no mutation or only mutations not associated with resistance (shown in grey around the map), or as yet uncharacterised mutations (shown in blue around the map) are detected, respectively. Results shown correspond to two isolates representative of two strain lineages distinguished by a) the absence (comprising seven isolates in total) or b) presence (five isolates in total) of the fabG1 G-27C single nucleotide polymorphism (SNP), distinct M. bovis spoligotype and/or MIRU-VNTR (mycobacterial interspersed repetitive unit-variable number tandem repeat) signatures, respectively. Green lines above gene names represent the reference sequences with coverage breadth $>95 \%$. Limits of detection (LOD) of potential heteroresistance (reflected by subpopulations of reads bearing a mutation), depending on the coverage depths over individual sequence positions, are indicated by grey (LOD 3\%) and orange zones (variable LOD $>3-80 \%$ ) above the reference sequences. Information on mycobacterial species identification, based on $h s p 65$ sequence best match, and genotype of M. tuberculosis complex strain, based on spoligotype and lineage-defining, phylogenetic SNP, are shown in the centre of the circle. AMI: amikacin; BDQ: bedaquiline; CAP: capreomycin; CFZ: clofazimine; EMB: ethambutol; ETH: ethionamide; FQ: fluoroquinolones; KAN: kanamycin; LIN: linezolid; INH: isoniazid; PZA: pyrazinamide; RIF: rifampin; SM: streptomycin; SIT: spoligotype international type.

observational study received the standard first-line TB treatment, none of them had a miliary or CNS localisation and they were all reported as cured or with a completed treatment according to WHO classification. Prolonged follow-up will be necessary to assess their risks of relapse.

Although this first extended, nationwide estimation in a non-high income country revealed a relatively low zoonosis prevalence among TB patients (3.4\%), our findings are aligned with global calls for appropriate diagnostics and treatment of these patients and TB control measures at the animal/human interface $[1,2]$.

Salam El Achkar ${ }^{1,2}$, Christine Demanche ${ }^{1,3}$, Marwan Osman $^{2}$, Rayane Rafei ${ }^{2}$, Mohamad Bachar Ismail $\oplus^{2}$, Cyril Gaudin $^{4}$, Stéphanie Duthoy ${ }^{4}$, Frédérique De Matos ${ }^{4}$, Hiam Yaacoub ${ }^{5}$, Claire Pinçon $\circledast^{3}$, Monzer Hamze $^{2}$ and Philip Supply ${ }^{1}$

${ }^{1}$ Université de Lille, CNRS, Inserm, CHU Lille, Institut Pasteur de Lille, U1019 - UMR 8204 - CIIL - Centre d'Infection et d'Immunité de Lille, Lille, France. ${ }^{2}$ Laboratoire Microbiologie Santé et Environnement (LMSE), EDST, FSP, Université Libanaise, Tripoli, Lebanon. ${ }^{3}$ Université de Lille, CHU Lille, EA 2694 - Santé publique: épidémiologie et qualité des soins and Faculté de Pharmacie, Lille, France. ${ }^{4}$ Genoscreen, Lille, France. ${ }^{5}$ National Tuberculosis Program, Ministry of Public Health, Tripoli, Lebanon.

Correspondence: Philip Supply, Université de Lille, CNRS, Inserm, CHU Lille, Institut Pasteur de Lille, U1019 - UMR 8204 - CIIL - Centre d'Infection et d'Immunité de Lille, 1 rue du Prof Calmette, F-59000 Lille, France.

E-mail: philip.supply@ibl.cnrs.fr

Received: 11 March 2019 | Accepted after revision: 17 Sept 2019

Acknowledgements: S. El Achkar was supported by a fellowship from Association AZM and Saadeh. The study also benefitted from support by a national TB programme in Lebanon, International Organization for Migration, TDR/ World Health Organization Eastern Mediterranean Region, Hamidi Medical Center in Tripoli, Lebanon, Ecole Doctorale en Sciences et Technologie (Université Libanaise), Centre Hospitalier Universitaire de Lille. Sara Amrieh, Taha Abdou, Mariam Yehya, Imane Darwish, and Clara Khairallah (LMSE, Lebanon) are gratefully thanked for technical assistance. 
Conflict of interest: S. El Achkar reports a $\mathrm{PhD}$ fellowship from Association AZM and Saadeh, logistical and administrative support from National TB program in Lebanon, International Organization for Migration, TDR/World Health Organization Eastern Mediterranean Region, Hamidi Medical Center in Tripoli, Lebanon, Ecole Doctorale en Sciences et Technologie (Université Libanaise), Centre Hospitalier Universitaire de Lille, logistical support from GenoScreen, during the conduct of the study. C. Demanche reports a PhD fellowship (to S. El Achkar) from Association AZM and Saadeh, logistical and administrative support from National TB program in Lebanon, International Organization for Migration, TDR/World Health Organization Eastern Mediterranean Region, Hamidi Medical Center in Tripoli, Lebanon, Ecole Doctorale en Sciences et Technologie (Université Libanaise), Centre Hospitalier Universitaire de Lille, during the conduct of the study. M. Osman reports a PhD fellowship (to S. El Achkar) from Association AZM and Saadeh, logistical and administrative support from National TB program in Lebanon, International Organization for Migration, TDR/World Health Organization Eastern Mediterranean Region, Hamidi Medical Center in Tripoli, Lebanon, Ecole Doctorale en Sciences et Technologie (Université Libanaise), Centre Hospitalier Universitaire de Lille, other from Genoscreen, during the conduct of the study. R. Rafei reports a PhD fellowship (to S. El Achkar) from Association AZM and Saadeh, logistical and administrative support from National TB program in Lebanon, International Organization for Migration, TDR/World Health Organization Eastern Mediterranean Region, Hamidi Medical Center in Tripoli, Lebanon, Ecole Doctorale en Sciences et Technologie (Université Libanaise), Centre Hospitalier Universitaire de Lille, other from Genoscreen, during the conduct of the study. M.B. Ismail reports a $\mathrm{PhD}$ fellowship (to S. El Achkar) from Association AZM and Saadeh, other from National TB program in Lebanon, International Organization for Migration, TDR/World Health Organization Eastern Mediterranean Region, Hamidi Medical Center in Tripoli, Lebanon, Ecole Doctorale en Sciences et Technologie (Université Libanaise), Centre Hospitalier Universitaire de Lille, other from Genoscreen, during the conduct of the study. C. Gaudin was an employee of Genoscreen, during the conduct of the study. S. Duthoy was an employee of Genoscreen, during the conduct of the study. F. De Matos was an employee of Genoscreen, during the conduct of the study. H. Yaacoub has nothing to disclose. C. Pinçon has nothing to disclose. M. Hamze has nothing to disclose. P. Supply reports a PhD fellowship (to S. El Achkar) from Association AZM and Saadeh, logistical and administrative support from National TB program in Lebanon, International Organization for Migration, TDR/World Health Organization Eastern Mediterranean Region, Hamidi Medical Center in Tripoli, Lebanon, Ecole Doctorale en Sciences et Technologie (Université Libanaise), Centre Hospitalier Universitaire de Lille, and consultancy fees from Genoscreen, during the conduct of the study.

Support statement: S. El Achkar was supported by a fellowship from Association AZM and Saadeh. Funding information for this article has been deposited with the Crossref Funder Registry.

\section{References}

World Health Organization (WHO). Roadmap for Zoonotic Tuberculosis. Geneva, WHO, 2017.

2 Olea-Popelka F, Muwonge A, Perera A, et al. Zoonotic tuberculosis in human beings caused by Mycobacterium bovis - a call for action. Lancet Infect Dis 2017; 17: e21-e25.

3 World Health Organization (WHO). Global Tuberculosis Report. Geneva, WHO, 2018.

4 Konno K, Feldmann FM, McDermott W. Pyrazinamide susceptibility and amidase activity of tubercle bacilli. Am Rev Respir Dis 1967; 95: 461-469.

5 Scorpio A, Zhang Y. Mutations in pncA, a gene encoding pyrazinamidase/nicotinamidase, cause resistance to the antituberculous drug pyrazinamide in tubercle bacillus. Nat Med 1996; 2: 662-667.

6 Majoor CJ, Magis-Escurra C, van Ingen J, et al. Epidemiology of Mycobacterium bovis disease in humans, the Netherlands, 1993-2007. Emerg Infect Dis 2011; 17: 457-463.

7 Allix-Béguec C, Fauville-Dufaux M, Supply P. Three-year population-based evaluation of standardized mycobacterial interspersed repetitive-unit-variable-number tandem-repeat typing of Mycobacterium tuberculosis. J Clin Microbiol 2008; 46: 1398-1406.

8 International Labour Organization. Intertwined - A Study of Employers of Migrant Domestic Workers in Lebanon. Geneva, International Labour Organization, 2016.

9 World Health Organization (WHO). Global Tuberculosis Report. Geneva, WHO, 2015.

10 Müller B, Dürr S, Alonso S, et al. Zoonotic Mycobacterium bovis-induced tuberculosis in humans. Emerg Infect Dis 2013; 19: 899-908.

11 Makhado NA, Matabane E, Faccin M, et al. Outbreak of multidrug-resistant tuberculosis in South Africa undetected by WHO-endorsed commercial tests: an observational study. Lancet Infect Dis 2018; 18: 1350-1359.

12 Supply P, Allix C, Lesjean S, et al. Proposal for standardization of optimized mycobacterial interspersed repetitive unit-variable-number tandem repeat typing of Mycobacterium tuberculosis. J Clin Microbiol 2006; 44: 4498-4510.

13 Weniger T, Krawczyk J, Supply P, et al. MIRU-VNTRplus: a web tool for polyphasic genotyping of Mycobacterium tuberculosis complex bacteria. Nucleic Acids Res 2010; 38: W326-W331.

14 Magnus K. Epidemiological basis of tuberculosis eradication. 3. Risk of pulmonary tuberculosis after human and bovine infection. Bull World Health Organ 1966; 35: 483-508.

15 Gonzalo-Asensio J, Malaga W, Pawlik A, et al. Evolutionary history of tuberculosis shaped by conserved mutations in the PhoPR virulence regulator. Proc Natl Acad Sci USA 2014; 111: 11491-11496. 